\title{
Breakthroughs in plant retrotranscriptome and mobilome characterization enabled by Nanopore sequencing
}

Kirov I. ${ }^{1,2 *}$, Merkulov P. ${ }^{1}$, Gvaramiya S. ${ }^{1}$, Omarov M. ${ }^{1}$, Dudnikov M. ${ }^{1,2}$, Kolganova E. ${ }^{1}$, Komakhin R. ${ }^{1}$, Divashuk M. ${ }^{1,2}$, Karlov G. ${ }^{1}$, Soloviev A. ${ }^{1}$

${ }^{1}$ All-Russia Research Institute of Agricultural Biotechnology, Moscow, Russia

${ }^{2}$ Kurchatov Genomic Center of ARRIAB, All-Russia Research Institute of Agricultural Biotechnology, Moscow, Russia

*email: kirovez@gmail.com

The significant part of the vast majority of plant genomes is occupied by mobile elements including DNA transposons and retrotransposons. These elements played a crucial role in plant evolution and the breeding of new crop varieties. However, their repetitive nature challenges their investigation using 'gene-centric' bioinformatics tools remaining current mobile element activity poorly studied. To fill in this gap, we used different Nanopore sequencing approaches (direct RNA, whole-genome and Cas9-targeted sequencing) to detect ongoing transcriptional (retrotranscriptome) and transpositional (mobilome) activity of retrotransposons (RTEs). We unravelled hundreds of RTE transcripts in different plant species expressed under non-stressed and stressed conditions and in different organs and developmental stages. In most of the plant species, RTEs produced different isoforms with higher expression of GAG encoding transcripts. Using Cas9-targeted Nanopore sequencing we were able to detect tens of recent insertions in Arabidopsis thaliana population and Aegilops taushii genomes demonstrating an unprecedented level of resolution and sensitivity for mobilome studies. We found that this approach is more cost-effective compared to whole-genome Nanopore sequencing. Moreover, we designed a pipeline for easy detection of RTE and T-DNA insertions from Nanopore data. Features and further perspectives of Nanopore sequencing for plant mobilome biology studies will be discussed.

Acknowledgements: The reported study was funded by RFBR, project number 20-3470032. 